\title{
Regímenes de riesgo en el mercado de acciones colombiano ${ }^{1}$
}

\section{Risk Regimes in the Colombian Stock Market}

\section{Regimes de risco no mercado de ações colombiano}

\section{Jorge Mario Uribe Gil ${ }^{2}$}

Docente e investigador de la Universidad del Valle, Cali, Colombia

jorge.uribe@correounivalle.edu.co

Recibido: 29/09/2014

Aprobado: $24 / 11 / 2015$

1 Integrante del Grupo de Macroeconomía Aplicada y Economía Financiera de la Universidad del Valle. El autor agradece el apoyo de la Universidad del Valle y del Centro de Investigaciones y Documentación Socioeconómica (CIDSE) a través de convocatoria interna de la Facultad 2014. Agradece también la asistencia de investigación de Alexander Páez Bedoya y la colaboración de Julián Fernández Mejía. Las opiniones expresadas en este documento son responsabilidad del autor y no comprometen a la Universidad del Valle.

2 Maestría en Economía, Master of Research in Economics. 


\title{
Resumen
}

En el presente artículo, se busca contrastar la hipótesis de posibles cambios en los niveles de riesgo del mercado de acciones colombiano. Se estima un AR-SWARCH para los retornos del Índice General de la Bolsa de Valores de Colombia durante el período julio 2001-diciembre 2013. Se encuentra que existen dos regímenes de riesgo. En aquel de mayor riesgo, el factor que mide cuánto se incrementa la varianza asciende a 2,23, lo cual subraya la necesidad de considerar este fenómeno de transición entre regímenes dentro de los análisis hechos por practicantes financieros y reguladores en el país. Estos regímenes se relacionan con fenómenos previamente detectados en la literatura como fallas en términos de eficiencia informacional (o burbujas) y cambios significativos en el tamaño y liquidez del mercado.

Palabras clave: SWARCH; IGBC; regímenes de riesgo; GARCH Colombia; volatilidad condicional.

Clasificación JEL: C58, Goo, G10, G11.

\begin{abstract}
In this paper, we seek to test the hypothesis of possible changes in risk levels in Colombian stock market. An AR-SWARCH model is estimated for the returns of the General Index of Colombia Stock Exchange during the period July 2001-December 2013. Two different risk regimes are identified in the analysis. At the highest risk, the factor that measures how variance increases amounts to 2.23. Those findings highlight the need to consider the transition dynamics between regimes within the analysis by financial practitioners and regulators, operating in Colombian stock market. Those regimes are also related to previously detected failures in the literature in terms of informational efficiency (bubbles), and to significant changes in the size and liquidity of the market.
\end{abstract}

Keywords: SWARCH; IGBC; risk regimes; GARCH Colombia; conditional volatility.

\section{Resumo}

No presente artigo se busca contrastar a hipótese de possíveis câmbios nos níveis de risco no mercado de ações colombiano. Se estima um AR-SWARCH para os retornos do Índice Geral da Bolsa de Valores da Colômbia, durante o período julho 2001-dezembro 2013. Evidencia-se que existem dois regimes de risco. No primeiro, de maior risco, o fator que mede quanto se incrementa a variância ascende a 2,23, salientando a necessidade de considerar este fenômeno de transição entre regimes dentro das análises feitas por praticantes financeiros e reguladores no país. Estes regimes relacionam-se com fenômenos previamente detectados na literatura como falhas em termos de eficiência informacional (ou bolhas) e mudanças significativas no tamanho e liquidez do mercado.

Palavras-chave: SWARCH; IGBC; Regimes de risco; GARCH Colômbia; volatilidade condicional. 


\section{Introducción}

El riesgo de mercado es uno de los temas de mayor relevancia en las economías de mercado actuales, en las cuales el papel de los mercados financieros como canalizadores de recursos y suavizadores del riesgo intertemporal y transversal de la inversión es fundamental. La medición adecuada de este riesgo es por tanto una necesidad insoslayable, sobre la cual se apoyan decisiones de inversión privadas y políticas de regulación públicas, que afectan el devenir del sistema económico como un todo. Este estudio busca caracterizar el riesgo de mercado encarnado en los retornos del Índice General de la Bolsa de Valores de Colombia (IGBC) de forma adecuada, a la vez que contrasta la hipótesis de posibles cambios en los niveles de riesgo enfrentados en la historia reciente del mercado de acciones colombiano.

Una práctica habitual para la medición del riesgo de mercado son los modelos de heterocedasticidad condicional autorregresiva (ARCH) propuestos por Engle (1982), y su versión generalizada, GARCH, introducidos por Bollerslev (1986), así como distintas derivaciones de los mismos ${ }^{3}$. Los anteriores constituyen una herramienta de uso común para la administración del riesgo financiero contemporánea en el mundo entero. En Colombia, particularmente, son utilizados de forma extensiva por reguladores como la Superintendencia Financiera o el Banco de la República, y por entidades interesadas, por norma o por políticas internas de gestión, en la administración del riesgo. Estos modelos son frecuentemente empleados, por ejemplo, para el cálculo de medidas de valor en riesgo (VeR) o pérdida esperada en las colas $s^{4}$.

No obstante, son muchos los trabajos que han llamado la atención sobre la posibilidad de estimar persistencias espurias de los choques, así como pronósticos condicionales pobres, cuando se trabaja dentro del contexto de los modelos de la familia GARCH. Estos problemas son teóricamente muy probables si las volatilidades "no condicionales" están sujetas a cambios de régimen en el período muestral que se utiliza para realizar las estimaciones, tal y como ha sido resaltado por Lamoureux y Lastrapes (1990).

Con el fin de abordar el problema de estos posibles cambios estructurales, Hamilton y Susmel (1994) proponen modelar en estos casos, de forma endógena, la probabilidad de transición entre distintos regímenes, dentro de cada uno de los cuales un modelo ARCH determinaría la evolución de la varianza condicional de la serie bajo estudio. La forma propuesta es mediante un modelo switching, como el planteado por Hamilton (1989), que gobernaría el régimen de la varianza no condicional, sobre la cual fluctuaría la serie de varianzas condicionales, que obedece a un proceso generador de datos del tipo ARCH (Engle 1982).

En este documento se estima un modelo ARCH con cambio de régimen (SWARCH) para el mercado de acciones colombiano, desde el día de la unificación de las tres bolsas que operaban en el país, el 3 de julio de 2001, hasta el 20 de diciembre de 2013. Lo anterior permite la identificación de distintos

3 Bollerslev (2008) provee un glosario sobre el tema.

4 Uribe y Ulloa (2011) hacen una revisión de las técnicas aplicadas a Colombia y a otros países de la región. 
regímenes de riesgo en el mercado, y ayuda en el avance de la comprensión de los determinantes de los "estados de riesgo" en este mercado.

El resto de este documento se organiza como sigue: en la primera sección se motiva el uso de los modelos SWARCH para el caso colombiano; en la segunda se presentan algunos documentos relacionados; en la tercera se expone el modelo propuesto por Hamilton y Susmel (1994); en la cuarta se consignan los principales resultados de la aplicación, identificando posibles vínculos teóricos con otros estudios que tratan sobre el mercado de acciones en el país, en términos de capitalización, liquidez, posibles ineficiencias informacionales y burbujas. La quinta sección concluye.

\section{Evolución reciente del mercado de acciones colombiano}

Existen varios indicios teóricos para considerar apropiado el modelo SWARCH de Hamilton-Susmel para modelar el comportamiento de la serie del Índice General de la Bolsa de Valores de Colombia. Este modelo permite poner a prueba una hipótesis sobre la existencia de distintos regímenes de riesgo en el mercado, que a su vez están asociados con estudios anteriores en la materia, sobre todo en términos de eficiencia informacional.

En el gráfico 1 se muestran el nivel del IGBC y sus retornos diarios para el período: 3 de julio de 2001 a 20 de diciembre de 2003.

Gráfico 1. (a) IGBC y (b) sus retornos logarítmicos diarios

(a)

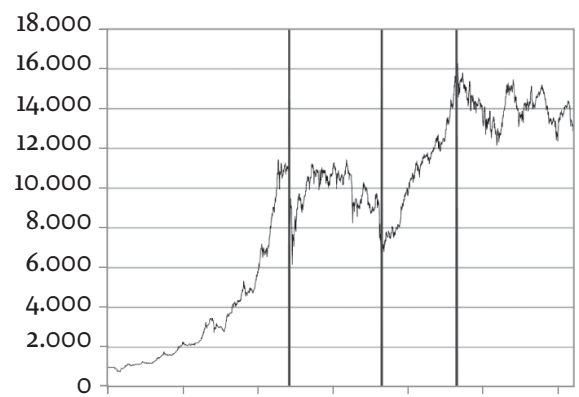

jul-01 jul-03 jul-05 jul-07 jul-09 jul-11 jul-13 (b)

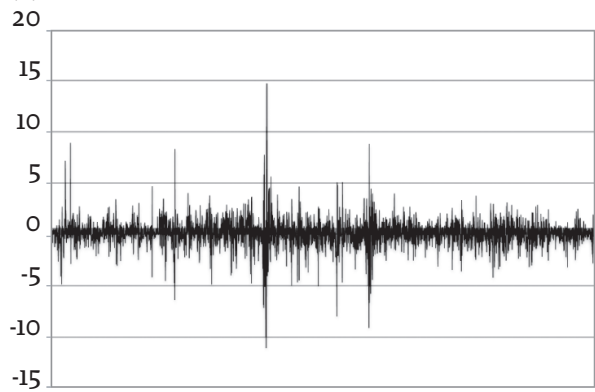

jul-01 jun-03 may-05 abr-07 mar-09 feb-11 ene-13

Nota: El IGBC está en puntos, los retornos en porcentaje.

Fuente: Bolsa de Valores de Colombia, calculos del autor.

En el panel (a) del gráfico 1 se observan cuatro tramos de comportamientos distintos en la evolución del índice colombiano: de julio de 2001 a mayo de 2006, un período de valorización prácticamente ininterrumpido; posteriormente, un período de relativa turbulencia en el mercado, de junio de 2006 a octubre de 2008, el cual no presenta una tendecia claramente definida. De noviembre de 2008 a septiembre de 2010, un intervalo de nueva valorización y, finalmente, de octubre de 2011 hasta 
diciembre de 2013, un tramo de relativa calma sin una tendecia perceptible en la evolución de la serie.

Los períodos, sencillamente caracterizados aquí, están asociados con momentos de mayor o menor eficiencia del mercado en el sentido débil, tal y como lo muestran Uribe y Ulloa (2011) y como se puede apreciar el gráfico 2. Por ejemplo, a pesar de que el primer y tercer períodos son de fuertes valorizaciones en el índice, en el primero se puede rechazar la hipótesis de caminata aleatoria como proceso generador de los datos, mientras que en el segundo esto no es posible, al utilizar razones de varianza (dinámicas) como las sugeridas por Lo y Mackinlay (1988). Los modelos SWARCH explorados en el presente contexto permitirán por su parte constatar si estos períodos de mayor o menor eficiencia informacional, se traslapan con distintos niveles de riesgo no condicionales de la serie de retornos del IGBC.

Gráfico 2. Indicador de eficiencia (razones de varianza), Colombia 2005-2010

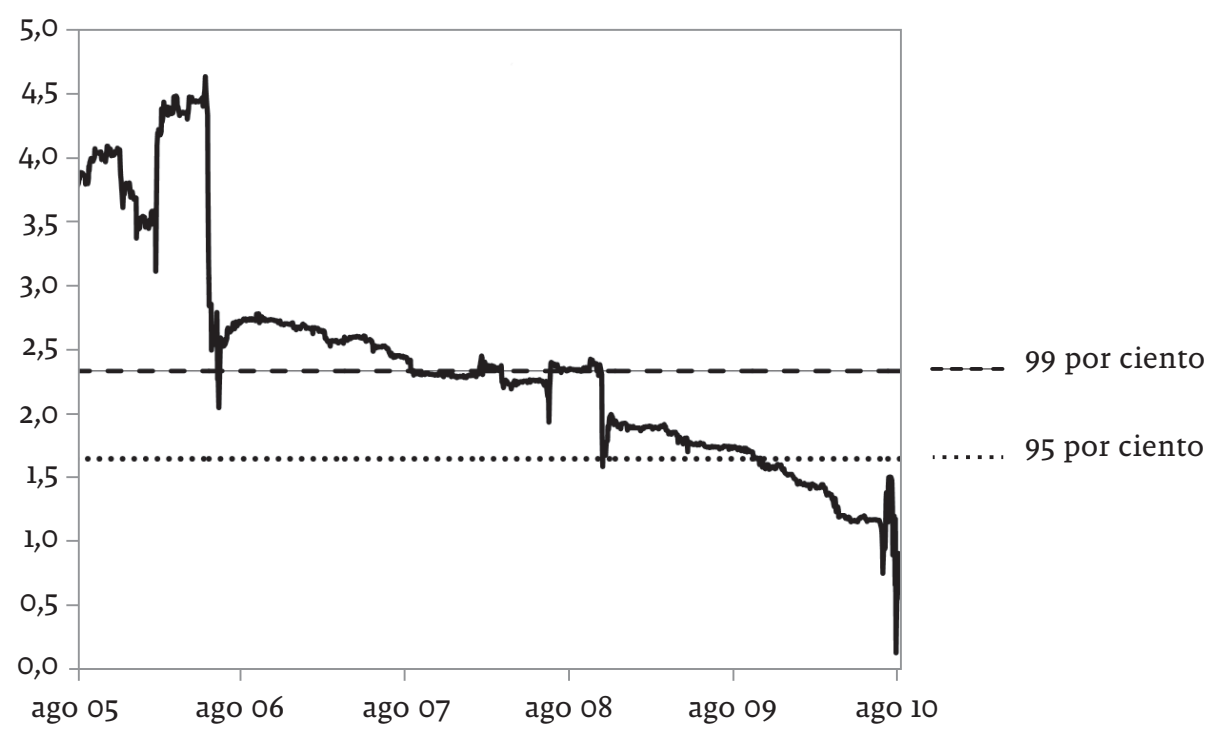

Fuente: Uribe y Ulloa (2011, 152).

Por otra parte, Uribe, Mosquera y Restrepo (2013) resaltan que durante este mismo período el mercado accionario colombiano ha sufrido fuertes cambios en cuanto a tamaño y liquidez, lo cual podría afectar la estabilidad del proceso generador de datos, en términos estadísticos, al inducir cambios estructurales de la media y la varianza condicionales o no condicionales de la serie de los retornos bursátiles (gráfico 3). Como respaldo a esta hipótesis se pueden citar los estudios de Bellalah y Nguyen (2008) y Nguyen (2008), quienes encuentran evidencia de

5 Cada punto es el estadístico de eficiencia propuesto por Lo y Mackinlay (1988), el cual debe ser igual a cero bajo la hipótesis nula de caminata aleatoria. El primer punto incluye los datos de julio de 2001 a julio de 2005 . Se presentan las bandas de confianza bajo la nula, al $99 \%$ y $95 \%$. 
cambios estructurales en los parámetros estimados que caracterizan los procesos estocásticos de las series financieras de varios mercados emergentes, después de procesos de liberalización financiera que involucran incrementos en los volúmenes transados y la capitalización de mercado, análogos a los experimentados en Colombia en años recientes, documentados por Uribe, Mosquera y Restrepo (2013).

Gráfico 3. Capitalización relativa suavizada (a), y volumen transado suavizado (b) de mercados de acciones latinoamericanos

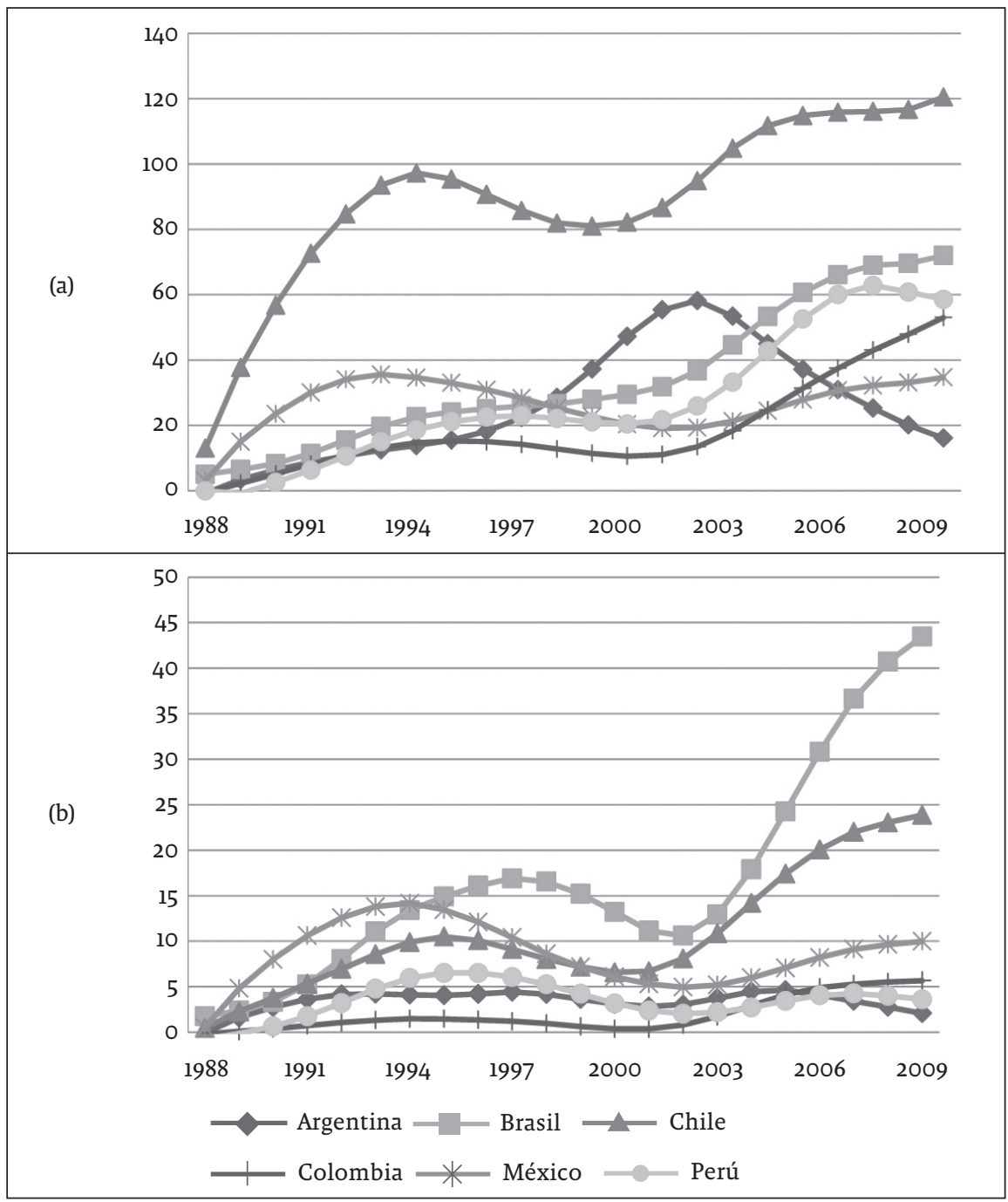

Fuente: Uribe, Mosquera y Restrepo (2013, 213-214).

En resumen, la importancia de la estimación de un modelo SWARCH para la modelación de la varianza condicional de los retornos del IGBC radica en que, dado el amplio uso de los modelos de la familia ARCH por parte de los agentes en el 
mercado financiero (e.g. bancos, comisionistas, fondos de pensiones) y de los reguladores (e.g. Superintendencia Financiera) para la estimación del riesgo, será conveniente introducir un modelo robusto ante cambios de regímenes y evitar análisis incorrectos sobre estimaciones de pudieran contener medidas de persistencia espuria y que, en general, podrían representar una pobre caracterización econométrica de las distribuciones condicionales y no condicionales de los retornos de la serie del IGBC, y por ende del riesgo de mercado.

Adicionalmente, es de resaltar que este modelo permite transiciones repetidas entre distintos regímenes, con sendas probabilidades de transición, lo cual es muy conveniente, dado que nada garantiza que la varianza condicional no fluctúe entre uno y otro estado, sin identificarse completamente con ninguna de las dos estimaciones por un periodo prolongado.

Finalmente, a un nivel más teórico se puede rescatar que el modelo SWARCH permitirá identificar distintos "regímenes de riesgo" en el mercado de acciones colombiano, lo cual puede ser útil por sí sólo para quienes diseñan la política económica, y a la vez para quienes administran fondos de inversión con posiciones en acciones locales. De esta forma, distintos regímenes de riesgo podrían justificar estrategias diversas como el trading activo, o la estrategia de comprar y mantener, según si existen indicios de un movimiento continuo entre distintos regímenes en el mercado, o si por el contrario los regímenes no condicionales parecen prolongarse durante amplios intervalos de tiempo.

\section{Literatura relacionada: riesgo cambiante en mercados emergentes}

Además de los estudios de Bellalah y Nguyen (2008) y Nguyen (2008), mencionados antes, que identifican cambios de régimen en las series que describen los precios de activos financieros en mercados latinoamericanos, es posible encontrar en la literatura varios trabajos que abordan el estudio de este fenómeno desde distintas perspectivas metodológicas y reportan resultados relacionados con los encontrados aquí.

Dentro de estos se pueden contar los estudios de Bekaert y Harvey (1997; 2000) quienes presentan una revisión completa de la literatura relacionada con la especulación internacional y su relación con los mercados emergentes de acciones, sobre todo en términos de riesgo. Tal estudio permite contrastar la evolución de la volatilidad en diferentes mercados, y entender algunas de las razones por las cuales esta podría ser distinta entre mercados, y en el mismo mercado después de cambios en las condiciones regulatorias, dando paso a distintos regímenes de volatilidad. También están los trabajos de Kim y Signal (2000), Cuñado, Gómez y Pérez (2006), quienes en líneas generales soportan la idea de que la liberalización de los mercados reduce los niveles de volatilidad del mercado, llevando a la aparición de varios regímenes en términos de riesgo. No obstante, Levine y Zerbos (1998), Stiglitz (2000) y Miles (2002), documentan un incremento en la volatilidad del mercado después de llevadas a cabo reformas que pueden ser clasificadas como de liberalización del mercado, o después de enfrentar choques idiosincráticos que afectan las series financieras (Aggarwal, Inclan y Leal 1999), sustentando indirectamente la necesidad teórica de abordar el fenómeno mediante modelos por regímenes de volatilidad para la compresión teórica del riesgo, pero sin ahondar en los aspectos econométricos de los 
mismos. Estudios como De Santis e Imrohoroglu (1997) o Jayasuriya (2005) muestran por su parte evidencia no concluyente que apoye alguna de las dos direcciones.

En Colombia el estudio de estos tópicos es escaso. Aunque sí existen abundantes escritos que abordan el diagnóstico de distintas facetas del mercado de capitales no intermediado, la mayoría de los cuales será referenciada en lo que queda del documento en la medida en la que se motive su introducción. En términos generales, se tiene el documento de Uribe (2007) que estima distintas medidas relacionadas con el riesgo, la eficiencia, la integración internacional y el riesgo del mercado de acciones colombiano y otros mercados de la región. Este estudio utiliza en la modelación del riesgo distintas especificaciones de modelos ARCH, GARCH y TARCH con el fin de caracterizar el riesgo de inversión financiera en Colombia y otros mercados, pero no explora la posibilidad de transición del mercado entre distintos regímenes de riesgo, tal y como se plantea en el presente documento.

Finalmente, en términos teóricos, se tiene que el estudio de Branch y Evans (2013), en el cual los agentes utilizan algoritmos de aprendizaje recursivo para fijar sus posiciones de portafolio óptimas, da lugar a un modelo por regímenes coherente con el modelo empírico que aquí se presenta.

\section{Modelo SWARCH}

El modelo SWARCH de Hamilton y Susmel (1994) es una mezcla entre los modelos ARCH de Engle (1982) y los modelos Switching de Hamilton (1989), la cual consiste básicamente en ponderar por factores distintos una estimación de la volatilidad condicional, obtenida mediante el uso de modelos $\mathrm{ARCH}$, dependiendo del régimen en el que se encuentre la variable. Los factores en dicha ponderación dependerán de estados no observables (regímenes) que gobiernan la trayectoria de la variable aleatoria, los cuales en general pueden ser descritos por una cadena de Markov. Considérese el modelo AR(p)-SWARCH-L(k,q) en [l]-[4]:

$$
\begin{aligned}
& r_{t}=\alpha+\phi_{1} r_{t-1}+\cdots+\phi_{p} r_{t-p}+u_{t} \\
& u_{t}=\sqrt{g_{s t}} \times \tilde{u}_{t} \\
& \tilde{u}_{t}=h_{t} \cdot v_{t} ; v_{t} \sim \text { i.i.d }(0,1) \\
& h_{t}^{2}=a_{0}+a_{1} \tilde{u}_{t-1}^{2}+a_{2} \tilde{u}_{t-1}^{2}+\cdots+a_{q} \tilde{u}_{t-q}^{2}+\xi \cdot d_{t-1} \cdot \tilde{u}_{t-1}^{2}, \\
& \text { donde } d_{t-1}=1 \text { si } \tilde{u}_{t-1} \leq 0 \text { y } d_{t-1}=0 \text { si } \tilde{u}_{t-1}>0
\end{aligned}
$$

La ecuación [1] corresponde a la media condicional, la cual sigue un proceso autorregresivo de orden $\mathrm{p}$. Los residuales de esta ecuación son una función de $g_{s t} \mathrm{Y}$ $\tilde{u}_{t}$, donde $g_{s t}$ es una constante que depende del régimen $\left(s_{t}\right)$ en el que se encuentre la variable $u_{t}$, y $\tilde{u}_{t}$ es una variable que sigue un proceso ARCH del tipo descrito en [3]-[4], con posibles efectos de apalancamiento (leverage) medidos por la variable indicadora $d_{t-1}$. 
La probabilidad de ubicarse en un régimen determinado estará gobernada por un proceso markoviano del tipo: $\operatorname{Prob}\left(s_{t}=j \mid s_{t-1}=i, s_{t \cdot 2}=k, \ldots, \tilde{u}_{t \cdot-1} \tilde{u}_{t-2} . ..\right)=\operatorname{Prob}\left(s_{t}=j \mid s_{t-1}=i\right)=p_{i j}$, para $\mathrm{i}, \mathrm{j}=1,2, \ldots K$ y matriz de transición de probabilidad dada por:

$$
\boldsymbol{P}=\left[\begin{array}{cccc}
p_{11} & p_{21} & \cdots & p_{K 1} \\
p_{12} & p_{21} & \cdots & p_{k 2} \\
\vdots & \vdots & \ddots & \vdots \\
p_{1 K} & p_{2 K} & \cdots & p_{K K}
\end{array}\right]
$$

En la cual cada columna en $\boldsymbol{P}$ suma 1.

La variable $\tilde{u}_{t}$ es multiplicada por la constante $\sqrt{ } g_{1}=1$ cuando el proceso se encuentra en el régimen representado por $s_{t}=1$, multiplicada por $\sqrt{ } g_{2}$ cuando $s_{t}=2, y$ en general por $\sqrt{ } g_{k}$ cuando $s_{t}=K$. El factor para el primer estado $g_{1}$ es normalizado a la unidad con $g_{j} \geq 1$ para $j=2,3, . . K$. El cambio en el régimen representará pues un cambio en la escala del proceso.

Por otra parte, las ecuaciones [3] y [4] representan un proceso ARCH (q) con efecto leverage análogo al sugerido por Glosten, Jagannathan y Runkle (1993), el cual busca capturar los efectos asimétricos que los choques positivos y negativos puedan tener sobre la varianza condicional. En este documento se hará $\xi=0$ con el fin de concentrar el análisis en los niveles de riesgo no condicionales, sin desviar la atención sobre posibles mejoras en la estimación condicional, importantes sobre todo en el proceso de conformación de portafolios óptimos en media-varianza por parte agentes involucrados en el mercado de forma activa.

El estimador utilizado para calcular los parámetros del modelo descrito en [1]-[4] es el de máxima verosimilitud, descrito en el apéndice de Hamilton y Susmel (1994). En términos generales el proceso consiste en evaluar la función de log de verosimilitud muestral, reiterativamente, hasta lograr un máximo global. Tal función está dada por:

$\mathcal{L}=\sum_{t=1}^{T} \ln f\left(r_{t} \mid r_{t-1}, r_{t-2}, \ldots, r_{t-T}\right)$

$\mathrm{Y}$ esta puede ser maximizada numéricamente con respecto a los parámetros del modelo, sujeta a las restricciones $\sqrt{ } g_{1}=1, \sum_{j=1}^{\mathrm{K}} p_{i j}=1$ para $i=1,2, \ldots K$ y $0 \leq p_{i j} \leq 1$ para $i, j=1,2, \ldots K$.

También es posible inferir el estado particular en el que el proceso estuvo en cada momento $t$. Esta inferencia se resume en:

i) "Probabilidades filtradas", las cuales indican la probabilidad de que el mercado se haya encontrado en uno de los $K$ distintos estados posibles en cada momento $t$, condicionando por la información revelada hasta el período anterior a la realización del estado, es decir hasta $(t-1)$.

ii) "Probabilidades suavizadas", las cuales indican la probabilidad de que el mercado se haya encontrado en uno de los $K$ distintos estados posibles en cada momento $t$, condicionando por la información total en la muestra. Estas segundas, dadas por:

$$
p\left(s_{t} \mid r_{T}, r_{T-1}, \ldots, r_{0}\right),
$$

Las cuales permitirán identificar los "niveles de riesgo" del mercado, ya que determinarán los niveles de la varianza no condicional sobre los cuales fluctúan las varianzas condicionales diarias, siguiendo la información contenida en [2]. 
Tanto las probabilidades filtradas, como las suavizadas suman uno entre los estados. Su longitud está asociada con el tamaño de la muestra efectiva, después de realizada la estimación, por tanto, para los fines de este documento se hace posible acceder a una estimación del régimen de riesgo (en principio no observado) en el cual se encontró el mercado en cada día de transacción desde el 3 de julio de 2001 hasta el 20 de diciembre de 2013. Es de esta forma como se posibilita la identificación de los "estados de riesgo" que hacen parte de los objetivos del estudio.

\section{Resultados}

En la tabla 1 se presentan los modelos estimados y los criterios de selección utilizados para elegir entre modelos. Un modelo $\operatorname{AR}(1)-S W A R C H(2,3)$, es decir, con un término autorregresivo en la media, tres términos ARCH en la varianza condicional y dos estados posibles; con errores distribuidos t-student presenta el mejor ajuste dentro de los seleccionados según lo criterios AIC (an information criterion) y BIC (Bayesian information criterion) modificados propuestos por Hamilton y Susmel (1994). Este ajuste también es mayor que el de un modelo AR(1)-GARCH (1,1) sin cambios de régimen, el cual presenta un log de verosimilitud menor $(-4.879,11)$. En el anexo 1 se presentan los coeficientes asociados con el modelo seleccionado.

Es posible identificar dos regímenes en el mercado de acciones colombiano: uno de "volatilidad alta" y uno de "volatilidad baja". En el primero, la volatilidad es aproximadamente 2,23 veces mayor que en el segundo, lo cual alerta sobre la necesidad de tener en cuenta los cambios dentro de la escala del proceso para la medición del riesgo de mercado en la práctica.

Un cambio tan marcado en el nivel de la serie significa que las varianzas condicionales estimadas con procesos GARCH tradicionales (con una sola varianza de convergencia no condicional, para toda la muestra) presentan parámetros de persistencia espurios y generan una subestimación del riesgo de mercado en un régimen y una sobreestimación del mismo en el otro. Se podría argumentar que este efecto es parcialmente mitigado por la naturaleza autorregresiva de los modelos GARCH, pero inevitablemente, este pobre ajuste entre regímenes llevará a una mala concepción del riesgo, y a una persistencia estimada de los choques mucho mayor de la que se reconcilia con los datos.

Una recomendación de sencilla implementación para los administradores de portafolio, o los reguladores, sería estimar dos modelos, seleccionando los datos para la estimación de cada modelo según el régimen al cual pertenezca cada uno. Y posteriormente utilizar un modelo u otro, dependiendo del régimen en el que se encuentre el mercado. Por supuesto, todavía faltaría pronosticar la pertenencia al régimen en cuestión, lo cual se puede hacer mediante la técnica propuesta en este documento o basándose en el conocimiento a priori del mercado que tenga el interesado. Es decir, las creencias subjetivas del administrador de portafolio sobre el régimen que esté operando en el mercado (alto o bajo). 
Tabla 1. Comparación de modelos ${ }^{6}$

\begin{tabular}{|c|c|c|c|c|c|c|c|c|}
\hline \multicolumn{6}{|c|}{ Log de verosimilitud } & \multicolumn{3}{|c|}{ Parámetros } \\
\hline Nombre & $A R C H$ & Régimen & $P h i=1$ & $P h i=2$ & $P h i=3$ & $P h i=1$ & $P h i=2$ & $P h i=3$ \\
\hline $\operatorname{SWARCH}(2,1)$ & 1 & 2 & $-4.799,6$ & $-4.799,6$ & $-4.798,5$ & 9 & 10 & 11 \\
\hline SWARCH $(2,2)$ & 2 & 2 & $-4.756,8$ & $-47.55,9$ & $-4.763,2$ & 10 & 11 & 12 \\
\hline $\operatorname{SWARCH}(2,3)$ & 3 & 2 & $-4.775,3$ & $-4.783,1$ & $-4.805,7$ & 11 & 12 & 13 \\
\hline $\operatorname{SWARCH}(3,1)$ & 1 & 3 & $-4.733,7$ & $-4.733,7$ & NA & 12 & 13 & 0 \\
\hline $\operatorname{SWARCH}(3,2)$ & 2 & 3 & $-4.716,3$ & $-4.748,0$ & NA & 13 & 14 & 0 \\
\hline t-SWARCH $(2,1)$ & 1 & 2 & $-4.728,3$ & $-4.728,0$ & $-4.727,7$ & 10 & 11 & 12 \\
\hline t-SWARCH $(2,2)$ & 2 & 2 & $-4.696,0$ & $-4.727,9$ & $-4.703,4$ & $\mathrm{ll}$ & 12 & 13 \\
\hline t-SWARCH $(2,3)$ & 3 & 2 & $-4.680,1$ & $-4.688,1$ & $-4.724,7$ & 12 & 13 & 14 \\
\hline t-SWARCH(3,1) & 1 & 3 & $\mathrm{NA}$ & $-4.727,6$ & $-4.746,3$ & 0 & 14 & 15 \\
\hline
\end{tabular}

\begin{tabular}{|c|c|r|r|r|r|r|r|r|}
\hline Nombre & ARCH & \multicolumn{3}{|c|}{ Régimen } & \multicolumn{1}{c|}{ AIC } & \multicolumn{3}{c|}{ BIC } \\
\hline SWARCH(2,1) & 1 & 2 & $-4.808,6$ & $-4.809,6$ & $-4.809,5$ & $-4.836,5$ & $-4.840,6$ & $-4.843,6$ \\
\hline SWARCH(2,2) & 2 & 2 & $-4.766,8$ & $-4.766,9$ & $-4.775,2$ & $-4.797,8$ & $-4.801,0$ & $-4.812,4$ \\
\hline SWARCH(2,3) & 3 & 2 & $-4.786,3$ & $-4.795,1$ & $-4.818,7$ & $-4.820,4$ & $-4.832,3$ & $-4.859,0$ \\
\hline SWARCH(3,1) & 1 & 3 & $-47.45,7$ & $-4.746,7$ & NA & $-4.782,9$ & $-4.787,0$ & NA \\
\hline SWARCH(3,2) & 2 & 3 & $-4.729,3$ & NA & NA & $-4.769,6$ & NA & NA \\
\hline t-SWARCH(2,1) & 1 & 2 & $-4.738,3$ & $-47.39,0$ & $-4.739,7$ & $-4.769,3$ & $-4.773,1$ & $-4.776,9$ \\
\hline t-SWARCH(2,2) & 2 & 2 & $-4.707,0$ & $-4.739,9$ & $-4.716,4$ & $-4.741,1$ & $-4.777,1$ & $-4.756,7$ \\
\hline t-SWARCH(2,3) & 3 & 2 & $-4.692,1$ & $-4.701,1$ & $-4.738,7$ & $-4.729,3$ & $-4.741,4$ & $-4.782,1$ \\
\hline t-SWARCH(3,1) & 1 & 3 & NA & $-4.741,6$ & $-4.761,3$ & NA & $-4.785,0$ & $-4.807,8$ \\
\hline
\end{tabular}

Nota: se reportan el logaritmo de la función de verosimilitud y los criterios de selección en cada modelo. Los nombres de las columnas hacen referencia a cuando parámetros autorregresivos se incluyen en cada modelo dentro de la parte AR del modelo.

Fuente: cálculos del autor.

En los gráficos 4 y 5 se presentan las probabilidades suavizadas asociadas con cada uno de los dos regímenes identificados y se le comparan con el nivel del IGBC. Como se puede observar, el régimen de volatilidad baja es menos frecuente que el de volatilidad alta y está principalmente concentrado al principio y final del período de análisis. Las mayores extensiones de este régimen se presentan en períodos como: julio-septiembre de 2001; marzo-mayo y agosto-octubre de 2002; febrero-mayo y agosto-diciembre de 2003; diciembre de 2009 a abril de 2010 y el período más prolongado (con breves incursiones dentro del régimen de volatilidad alta) desde noviembre de 2012 hasta mayo de 2013. También este

6 En ocasiones el algoritmo de optimización utilizado por el programa GAUSS no permitió acceder a un candidato sólido de máximo global. No se reportan tales resultados, sino que en su lugar se reporta "NA", con el fin de seleccionar el modelo estimado dentro de los candidatos factibles. Todos los cálculos se llevaron a cabo usando la rutina provista por Hamilton, el autor original del modelo, en su página web: http://econweb.ucsd.edu/ jhamilton/. 
régimen describe mayoritariamente al período comprendido entre agosto y diciembre de 2013.

Gráfico 4. Probabilidades suavizadas de los regímenes de volatilidad baja (a) y alta (b)

(a)

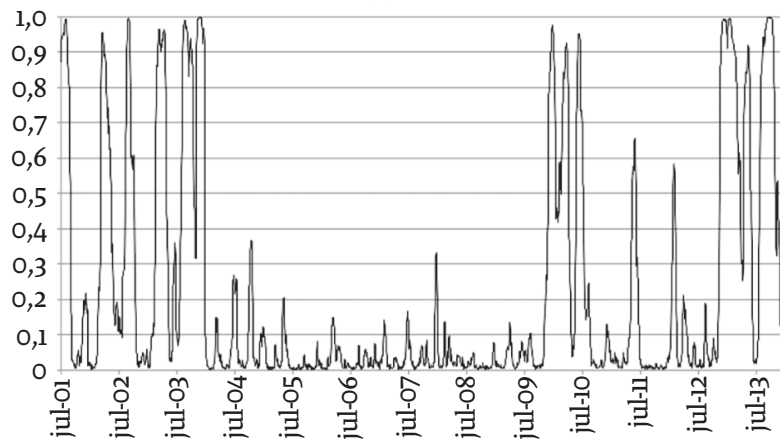

(b)

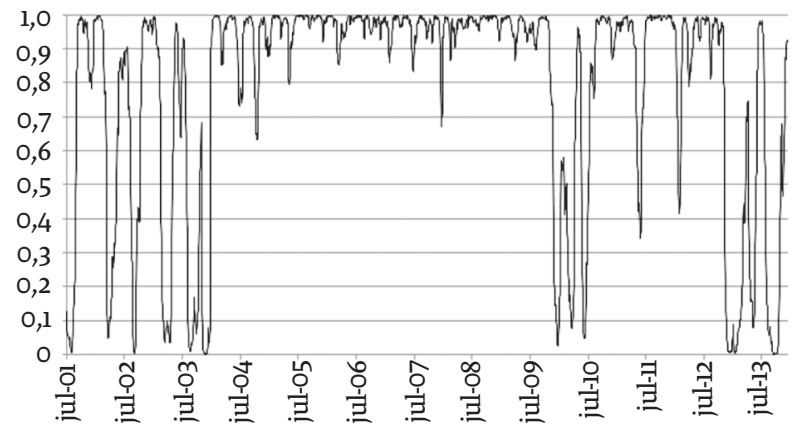

Fuente: Cálculos del autor.

Gráfico 5. Probabilidad suavizada de volatilidad baja (izquierda) e IGBC en niveles (derecha)

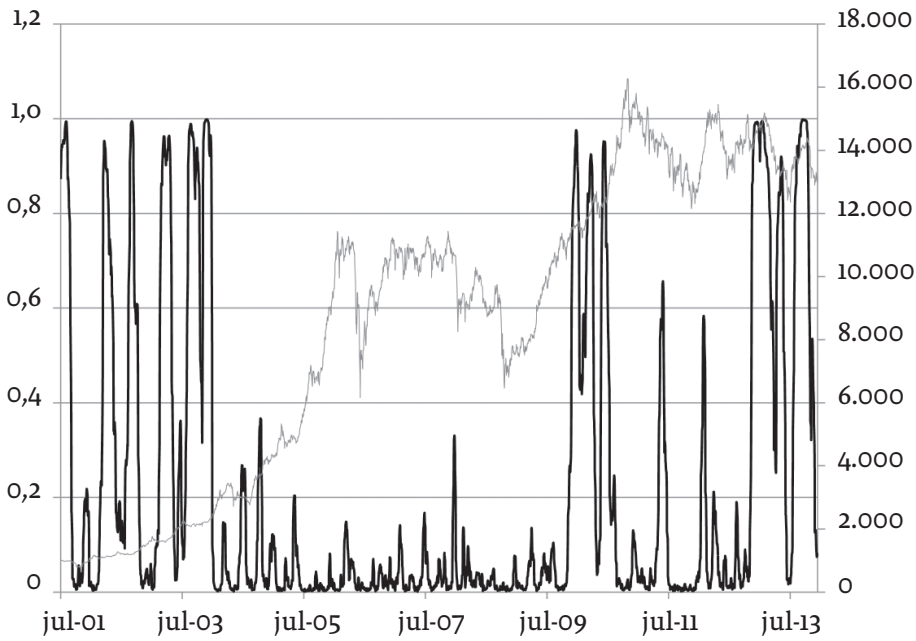

Fuente: Cálculos del autor. 
Con excepción de los períodos anteriores, y con notable presencia en el rango medio de la muestra que va desde enero de 2004 hasta noviembre de 2009, es el régimen de volatilidad alta el que mejor caracteriza la dinámica del mercado de acciones colombiano.

Es de notar también que existe un alto grado de concordancia entre los períodos de "burbuja" identificados por Uribe y Fernández (2014) identificados en el gráfico 6, y los períodos de "volatilidad alta". Durante los intervalos de burbuja la serie del IGBC no parece seguir una caminata aleatoria, tal y como se esperaría de un modelo tradicional de valoración de activos, sino un proceso explosivo que se asocia con la ruptura de la condición de eficiencia informacional y la aparición de estrategias especulativas ante el rompimiento de la condición de arbitraje.

Gráfico 6. Períodos de sobrevaloración resaltados con gris

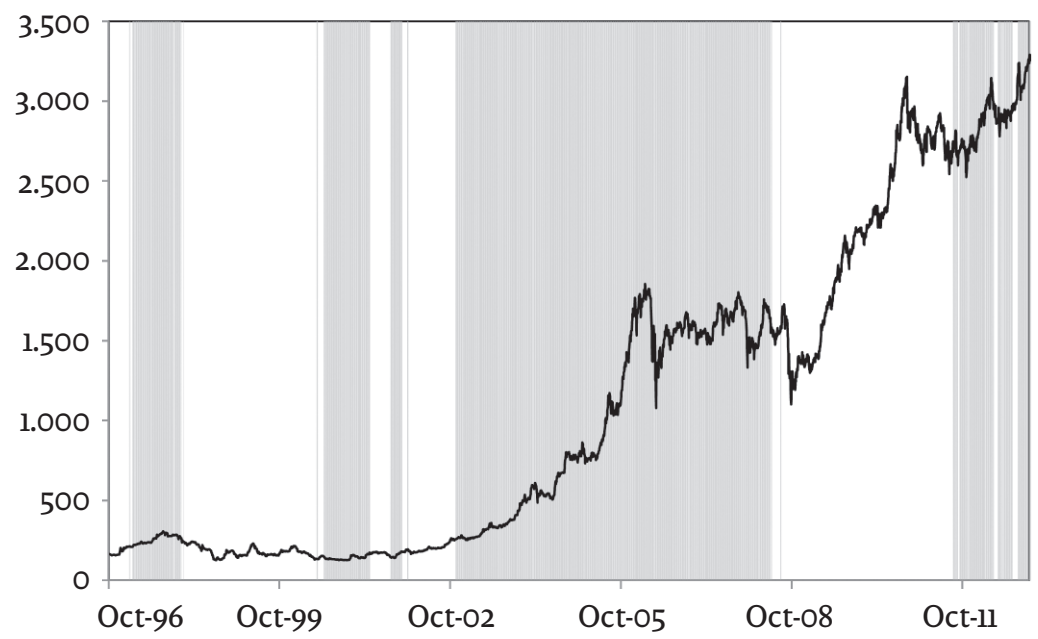

Fuente: Uribe y Fernández (2014).

Lo anterior parece indicar que el análisis de los segundos momentos arroja información complementaria sobre la dinámica de la serie en media, cuando se trata del mercado de acciones colombiano. Es de esta forma como se tiene entonces que las dinámicas de mayor valorización del mercado (y también de mayor corrección del mercado, como las posteriores a mayo de 2006) se pueden enmarcar en períodos que no se rigen por una caminata aleatoria, es decir de burbuja según Uribe y Fernández (2014) y al mismo tiempo, como procesos de alta volatilidad no condicional, es decir de alto riesgo, según los resultados del presente estudio. De la misma forma, los regímenes de volatilidad se relacionan con períodos de ineficiencia en el mercado (gráfico 1). Al estar construido a través de razones de varianza, el estadístico de eficiencia se puede ver directamente afectado por cambios de régimen como los aquí identificados en la volatilidad no condicional de la serie.

Ambas situaciones previenen al inversionista y al regulador sobre la necesidad de considerar dos modelos para el cálculo de sus relaciones riesgo-retorno, con el fin de optimizar sus gestión del riesgo financiero (o un modelo como el presente que abarque ambos casos). 


\section{Conclusiones e investigación futura}

Existen indicios teóricos y evidencia estadística suficientes como para afirmar que en el mercado de acciones colombiano han operado dos regímenes de volatilidad distintos, claramente identificables. Las estimaciones de riesgo de mercado que se hacen actualmente en Colombia, tanto a cargo del regulador como de los regulados, deberían tener en cuenta su existencia para tratar de aproximar el riesgo de la mejor manera posible. Es decir, se debería utilizar un modelo en media-varianza para construir estadísticos como el VeR, partiendo la muestra en dos regímenes como los identificados aquí y con base en ellos refinar los análisis de riesgo-retorno que se llevan a cabo en la actualidad.

Los distintos regímenes de volatilidad parecen tener relación con los períodos de burbujas o comportamientos explosivos en el mercado de acciones colombiano, cuando se les mide como en Uribe y Fernández (2014); o cuando se les compara con los resultados de estudios como el de Uribe y Ulloa (2011). En el primer caso, el período de burbuja es identificado por esos autores unos meses antes de que comience el régimen de alto riesgo que se presenta en este documento. Lo anterior pude indicar que el inflamiento de la burbuja no afecta los niveles de varianza (o riesgo), sino hasta que los agentes incorporan la posibilidad de tal burbuja dentro de la formación de sus expectativas. De la misma forma, el régimen de volatilidad alta termina meses después de que la burbuja se colapsa, y esto puede deberse, nuevamente, a cierto rezago en la formación de las expectativas de los agentes que operan en el mercado, los cuales podrían tardar algunos meses en incorporar dentro de sus funciones de reacción óptimas, la información sobre el colapso de la burbuja, y la consecuente mayor cercanía del precio y sus fundamentales. Esto se puede interpretar como evidencia débil a favor de los modelos teóricos que suponen agentes que aprenden, en formas recursivas o bayesianas. Branch y Evans (2013) construyen un modelo sobre burbujas y colapsos financieros, coherente con los hallazgos del presente estudio.

Es deseable profundizar en la identificación de los cambios en los fundamentales, o en la manera de percibir el precio de los activos por parte de los agentes, que están relacionados con estos cambios de régimen. Siguiendo los hallazgos de Uribe, Mosquera y Restrepo (2013), estos regímenes presentan una asociación (no identificada formalmente en este documento) con fenómenos como: un incremento pronunciado que se presenta en la capitalización bursátil de 2004 a 2009; un aumento en la razón de volumen transado como proporción del PIB de 2001 a 2006; un incremento en los flujos de entrada de la cuenta capitales (de 2003 a 2007), y un aumento sostenido en la participación dentro de su portafolio, de los títulos en renta variable, por parte de las Administradoras de Fondos de Pensiones (información de 2005 en adelante). Como se argumenta, todos estos fenómenos ocurren simultáneamente con el régimen de mayor riesgo aquí identificado, principalmente comprendido entre enero de 2004 y diciembre de 2009.

El anterior análisis ayuda a tejer una narrativa coherente sobre la historia reciente del mercado de acciones en Colombia, según la cual, este ha experimentado dinámicas importantes, en épocas recientes, en términos de capitalización y en menor medida liquidez; este proceso ha estado acompañado por mayores 
concentraciones de la oferta de títulos y ha estado impulsado, no por dinámicas de los fundamentales internos o externos (tasas de interés, nivel de precios, términos de intercambio, etc.) sino por presiones por el lado de la demanda (flujos de capitales de portafolios y compras de títulos por parte de las AFP). Tales dinámicas se tradujeron en valorizaciones del IGBC nunca observadas antes, desde 2004 a 2006, con correcciones importantes del mercado de 2006 a 2009. Este período estuvo dominado por dinámicas especulativas (burbujas, o ineficiencia informacional, donde los fundamentales no juegan un papel importante) y según los hallazgos de este estudio, también por mayores niveles de riesgo. El anterior constituye uno de los principales aportes a la literatura que hace este documento.

Se pensaría que es de esta forma como los "ciclos de riesgo" del mercado están relacionados con una percepción generalizada de la existencia de una sobrevaloración de los activos financieros en ciertos momentos, y de temores crecientes ante una eventual restricción de la política monetaria en varios momentos que la muestra alcanza a capturar.

Durante todo el período de la crisis global el régimen de volatilidad que operó en el mercado fue el alto. Esto puede deberse a factores fundamentales como las relaciones con economías muy afectadas como la de Estados Unidos, o a la presencia de empresas como Ecopetrol a partir del 2007 en la Bolsa de Valores, las cuales dependen en gran medida de commodities, en particular del petróleo, cuyo mercado internacional sufrió su propia turbulencia en el 2008.

En cualquier caso, el régimen no parece estar sincronizado con factores fundamentales externos claramente identificables y, en cambio, sí obedecer a factores idiosincráticos como los mencionados antes. Por ejemplo, durante el 2009 el régimen que operó fue el de volatilidad baja, aun cuando la turbulencia externa persistía, y solo se dio paso a un nuevo cambio en el 2010, en el cual se encontraría el mercado a la fecha en que es escrito este documento.

\section{Referencias bibliográficas}

Aggarwal, Reena, Carla Inclan y Ricardo Leal. «Volatility in Emerging Stock Markets». Journal of Financial and Quantitative Analysis, Vol. 34, 1999: 33-35.

Bekaert, Geert y Harvey Campbell. «Emerging Equity Market Volatility». Journal of Financial Economics, Vol. 43 (junio 1997): 29-78.

Bekaert, Geert y Harvey Campbell. «Foreign Speculators and Emerging Equity Markets». Journal of Finance, Vol. 55, n 2 (abril 2000): 565-613.

Bellalah, Mondher y Duc Nguyen. «Stock Market Liberalization, Structural Breaks and Dynamic Changes in Emerging Market Volatility". Review of Accounting Finance, Vol. 7, $\mathrm{n}^{\circ}$ 4, 2008: 396-411.

Bollerslev, Tim. «Generalized Autoregressive Conditional Heteroskedasticity». Journal of Econometrics, Vol. 31 (febrero 1986): 307-327.

Bollerslev, Tim. «Glossary to ARCH (GARCH)». CREATES Research Paper, $\mathrm{n}^{\circ}$ 200849, (septiembre 2008): 1-42.

Branch, William y George Evans. «Bubbles, Crashes and Risk». Economic Letters, Vol. 120, n 2, 2013: 254-258. 
Cuñado, Juncal, Javier Gómez y Fernando Pérez. «Changes in the Dynamic Behavior of Emerging Market Volatility: Revisiting the Effects of the Financial Liberalization». Emergind Markets Review, Vol. 7, 2006: 261-278.

De Santis, Giorgio y Selahattin Imrohoroglu. «Stock Returns and Volatility in Emerging Financial Markets». Journal of International Money and Finance, Vol. 16, n 4, 1997: 561-579.

Engle, Robert. «Autoregressive Conditional Heteroscedasticity with Estimates of the Variance of United Kingdom Inflation». Econométrica, Vol. 50, $\mathrm{n}^{\circ} 1$ (julio 1982): 987-1008.

Glosten, Lawrence, Ravi Jagannathan y David Runkle. «On the Relation Between the Expected Value and the Volatility of the Nominal Excess Return on Stocks». Journal of Finance, Vol. 48, n 5 (diciembre 1993): 1779-1801.

Hamilton, James. "A New Approach to the Economic Analysis of Nonstationary Time Series and the Business Cycle». Econométrica, Vol. 57, n 2 (marzo 1989): 357-384.

Hamilton, James y Raúl Susmel. «Autoregressive Conditional Heteroskedasticity and Changes in Regimens». Journal of Econometrics, Vol. 64 (septiembre 1994): 307-333.

Jayasuriya, Shamila. «Stock Market Liberalization and Volatility in the Presence of Favorable Market Characteristics and Institutions». Emerging Markets Review, Vol. 6, $\mathrm{n}^{\circ} 2$ (junio 2005): 170-191.

Kim, Han y Vijay Signal. «Stock Market Openings: Experience of Emerging Economies». Journal of Business, Vol. 73, $\mathrm{n}^{\circ}$ 1, 2000: 25-66.

Lamoureux, Christopher y William Lastrapes. «Persistence in Variance, Structural Change and the GARCH Model». Journal of Business and Economic Statistics, Vol. 8, n² 2 (abril 1990): 225-234.

Levine, Ross y Sara Zervos. "Capital Market Liberalization and Stock Market Development». World Development, Vol. 26, 1998: 1169-1183.

Lo, Andrew y Craig Mackinlay. «Stock Market Prices do not Follow Random Walks: Evidence from a Simple Specification Test». Review of Financial Studies, Society for Financial Studies, Vol. 1, nº 1 (primavera 1988): 41-66.

Miles, William. «Financial Deregulation and Volatility in Emerging Equity Markets». Journal of Economic Development, Vol. 27, n 2 (diciembre 2002): 113-126.

Nguyen, Duc. «An Empirical Analysis of Structural Changes in Emerging Market Volatility». Economics Bulletin, Vol. 6, nº 10 (marzo 2008): 1-10.

Stiglitz, Joseph. «Capital Market Liberalization, Economic Growth, and Instability». World Development, Vol. 28, nº 6 (junio 2000): 1075-1086.

Uribe, Jorge. "Caracterización del mercado accionario colombiano, 2001-2006: un análisis comparativo». Borradores de Economía (Banco de la República), $\mathrm{n}^{\circ}$ 456, 2007: 1-35.

Uribe, Jorge y Julián Fernández. «Burbujas especulativas en los mercados de acciones en América Latina». Lecturas de Economía, nº 81 (julio-diciembre 2014): 57-90.

Uribe, Jorge, Stephania Mosquera y Natalia Restrepo. «Mercado de acciones colombiano. Determinantes macroeconómicos y papel de las AFP». Sociedad y economía, $\mathrm{n}^{\circ} 24$ (junio 2013): 207-229. 
Uribe, Jorge e Inés Ulloa. «Revisando la hipótesis de los mercados eficientes: nuevos datos, nueva crisis, nuevas estimaciones». Cuadernos de Economía, Vol. 30, $\mathrm{n}^{\circ} 55$ (junio 2011): 127-154.

\section{Anexo 1. Estimaciones del modelo seleccionado}

\begin{tabular}{lcccc}
\hline Valor del log de verosimilitud & $4.680,1$ & & & \\
Constante & 0,08 & & & \\
Coeficiente AR(1) & 0,15 & & 0,10 & \\
Constante en la varianza & 0,18 & & 2,23 \\
Coeficientes ARCH(1) ARCH(2) ARCH(3) & 0,21 & 0,23 & 4,97 & \\
Factores de varianzas en cada régimen y & 2,00 & & \\
factor de escala & & 0,96 & 0,01 & \\
Probabilidades de transición de Markov & 0,04 & 0,99 & \\
& 4,83 & & \\
Grados de libertad de la distribución t &
\end{tabular}

Fuente: Elaboración del autor. 